\title{
Comparing the physical parameters of the intermediate and long GRB optical afterglows
}

\author{
József Kóbori $^{* a}$, Zsolt Bagoly ${ }^{a b}$, Lajos G. Balázs ${ }^{c d}$, István Horváth ${ }^{b}$, Dorottya \\ Szécsi ${ }^{a}$ \\ ${ }^{a}$ Dept. of Physics of Complex Systems, Eötvös University, H-1117 Budapest, Pázmány P. s. 1/A, \\ Hungary \\ ${ }^{b}$ Dept. of Physics, Bolyai Military University, H-1581 Budapest, POB 15, Hungary \\ ${ }^{c}$ Dept. of Astronomy, Eötvös University, H-1117 Budapest, Pázmány P. s. 1/A, Hungary \\ ${ }^{d}$ MTA CSFK Konkoly Observatory, H-1525 Budapest, POB 67, Hungary \\ E-mail: jkobori@caesar.elte.hu
}

\begin{abstract}
We compared the physical parameters (e.g. temporal decay index, spectral index) of the optical afterglows of intermediate and long GRBs. The GRBs were chosen from the sample used in Veres et al., ApJ, 725, pp. 1955-1964, 2010. Both data set of the long and intermediate GRB afterglows consist the same number of afterglows, and the redshift distributions are the same as well. The afterglow data was taken from the Swift UVOT archive system, and the data reduction was carried out using the Heasoft, version 6.11.
\end{abstract}

Gamma-Ray Bursts 2012 Conference-GRB2012,

May 07-11, 2012

Munich, Germany

\footnotetext{
${ }^{*}$ Speaker.
} 


\section{Data reduction - methods}

We analyzed the UVOT data of 20 gamma-ray burst afterglows. The GRBs were taken from the sample used in [1]. The authors used basically two methods for clustering the GRBs: the model based clustering (Bayesian Information Criterion method) and non-parametrical (K-meansand hierarchial) clustering. They found, that all of the methods give three components on the hardness-duration plane. To determine the membership of a given burst, they give the probability that a burst belongs to a group. For the details of the method please see [1].

This sample consists 46 intermediate and 331 long GRBs. From the former group 15 has UVOT detections, and 9 is bright enough to construct the light curves. The afterglows of long GRBs were chosen so that the redshift distributions of the two groups are the same. To determine the count rates we used the software Heasoft, version 6.11, with the CALDB version 20110731.

Since at some afterglows a color evolution can be observed, we do not normalized the count rates to the $\mathrm{V}$ filter, as it is a common practice in the literature. The count rates were first corrected for the Galactical extinction using the extinction maps constructed by Schlegel et. al([2]), then they were converted into $\mathrm{AB}$ fluxes. After that the lightcurves were fitted with a power-law function, and if a break was presented, than the two intervals were fitted separately.

Spectral energy distributions were constructed at arbitrarily chosen epochs, but if color evolution occured during the afterglow, the spectral slope was taken at the earliest epoch.

We also tried to determine the host extinction using the extinction curves described in [3], but probably because of the low number of the filters (just V, B and U), in most cases we got negative $A_{V}$ values.

\section{Optical vs. X-ray decay rates}

We compared the optical decay rates and the X-ray decay rates (Figure 1). In the case of the afterglows of the intermediate GRBs we found, that optical temporal indices are smaller than X-ray temporal indices, i.e. the X-ray afterglows decay faster than optical afterglows.

In contrast to the afterglows of intermediate GRBs, the decay rates for the optical and X-ray afterglows of long GRBs are approximately the same.

\section{Temporal decay index vs. redshift}

We investigated whether there is a connection between the optical temporal slope and redshift (Figure 2, right side). The result is interesting, because the decay rates of afterglows of long GRBs are weakly correlating with the bursts' redshift, as it can be seen on Figure 2, right side. Unfortunately, due to the low number of bursts in the sample, we can not test whether this correlation is significant or not, but, based on the literature, we conclude, that this correlation is only coincidental or due to the low number of bursts in the sample. 

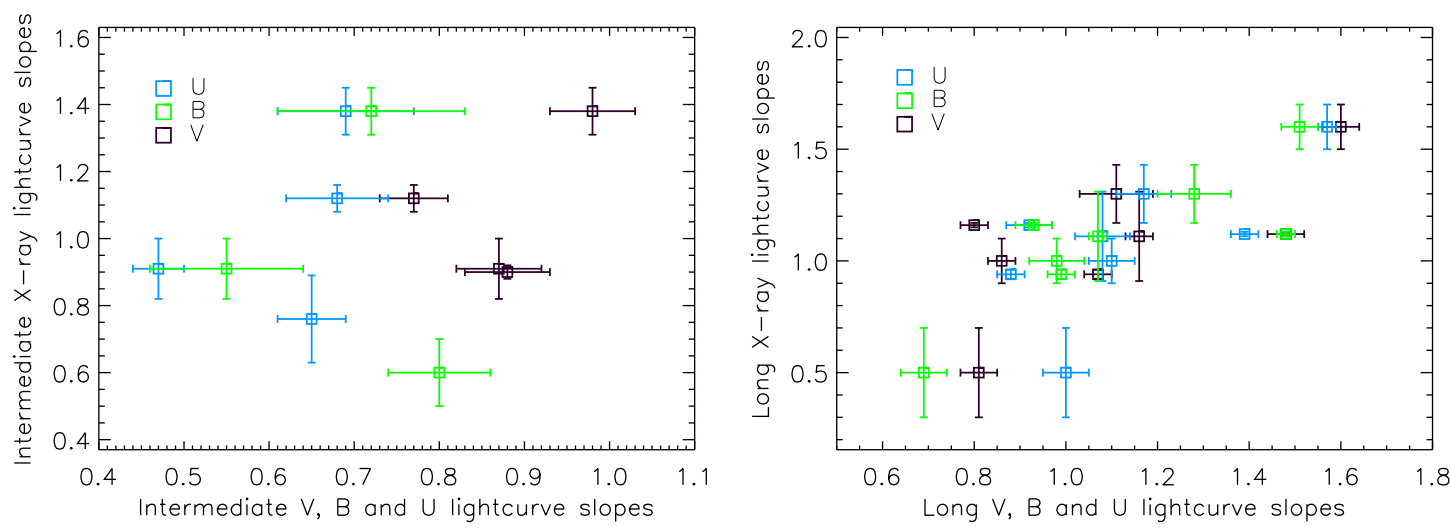

Figure 1: The left figure shows the X-ray lightcurve slopes of the intermediate GRBs plotted against the V, $\mathrm{B}$ and $\mathrm{U}$ lightcurve slopes, while on the right figure the X-ray and optical slopes are compared in the case of long bursts. From the plot it is obvious that the X-ray afterglows of the intermediate group decay faster than optical afterglows.

\section{Luminosity lightcurves}

The flux lightcurves were converted into luminosity lightcurves (Figure 2, left side) through $L(v)=\frac{4 \pi d_{L}^{2}}{(1+z)^{1-\beta_{o}}} F(v)$, where $v_{0}$ is the central frequency of the photometric filter, $d_{L}$ is the luminosity distance (with the following cosmological constants: $H_{0}=71 \mathrm{~km} \mathrm{~s}^{-1} \mathrm{Mpc}^{-1}, \Omega_{M}=0.27, \Omega_{\Lambda}=$ $0.73)$ and $\beta_{o}$ is the unabsorbed spectral index.
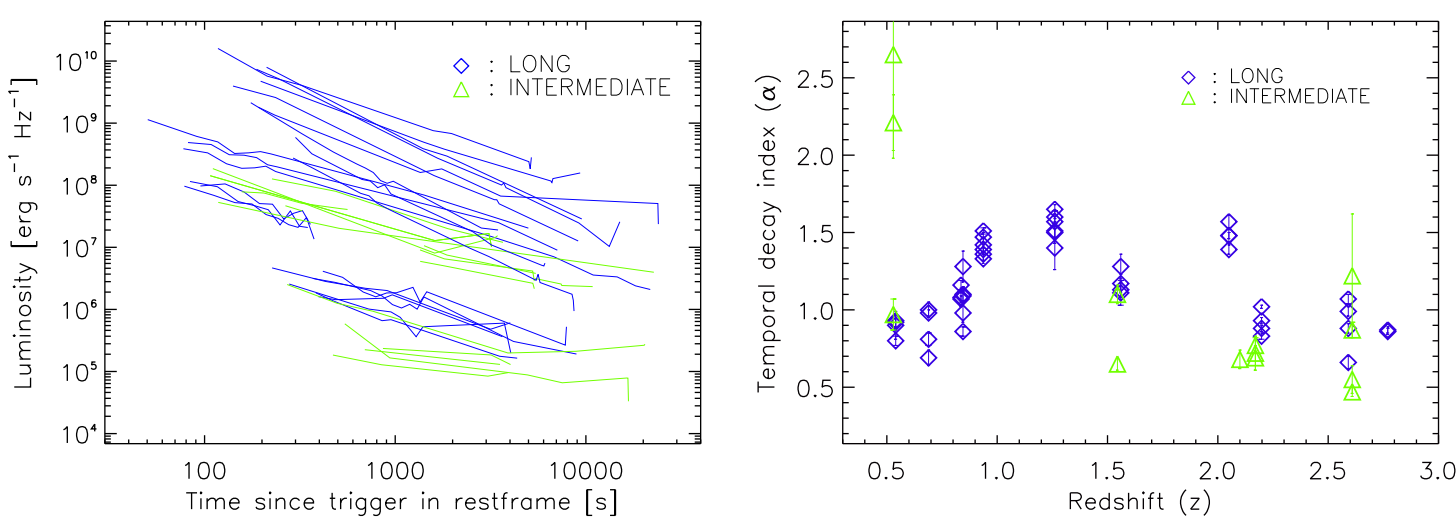

Figure 2: On the left figure the luminosity lightcurves can be seen. The blue lines indicate the V, B, U lightcurves of the long group, while the green lines mark the same filters, but of the intermediate group. The right figure shows the $\mathrm{V}, \mathrm{B}, \mathrm{U}, \mathrm{W} 1, \mathrm{M} 2$ and $\mathrm{W} 2$ lightcurve slopes plotted against the redshift. In the case of afterglows of intermediate GRBs no correlation can be discovered between the parameters, but contrarily at afterglows of the long group it seems, that there is a weak correlation between the redshift and optical decay rate. 


\begin{tabular}{lccrrccc}
\hline Long & $\beta$ & $\beta$ & Int. & & Filter & Long & Int. \\
\hline 060512 & 1.78 & 0.72 & 050801 & & $\bar{\alpha}_{V}$ & $1.10 \pm 0.02$ & $0.87 \pm 0.02$ \\
070518 & 1.8 & 1.28 & $050922 \mathrm{C}$ & & $\bar{\alpha}_{B}$ & $1.11 \pm 0.02$ & $0.69 \pm 0.05$ \\
$070810 \mathrm{~A}$ & 1.69 & 2.16 & 061007 & & $\bar{\alpha}_{U}$ & $1.12 \pm 0.02$ & $0.62 \pm 0.16$ \\
080330 & 0.54 & 3.19 & 080721 & & $\bar{\alpha}_{W 1}$ & $1.13 \pm 0.02$ & $1.48 \pm 0.2$ \\
080520 & 3.95 & 2.13 & $080916 \mathrm{~A}$ & & $\bar{\alpha}_{M 2}$ & $1.50 \pm 0.07$ & $2.21 \pm 0.18$ \\
$081007 \mathrm{~A}$ & 1.53 & 2.26 & $081203 \mathrm{~A}$ & & $\bar{\alpha}_{W 2}$ & $1.27 \pm 0.07$ & - \\
090426 & 1.9 & 1.89 & 090426 & &
\end{tabular}

Table 1: In the table the spectral and temporal decay indices are compared. Most of the spectral indices has an ordinary value, except in the cases of the GRB 080520 and GRB 080330, which are relatively high and low, respectively.

\section{Temporal and spectral indices}

Comparing the average temporal indices of the two group (Table 1, right side), we can see, that the decay slopes of afterglows of long GRBs are clustering around 1.1-1.2, while in the case of the intermediate GRBS the scatter is big, but the trend is, that the shorter the wavelength, the greater the decay index.

The spectral indices (Table 1, left side) found for the two groups are typical in the standard modell, there is no difference between the groups.

\section{Acknowledgements:}

This work was supported by the Hungarian OTKA-77795 grant.

This research has made use of data obtained through the High Energy Astrophysics Science Archive Research Center Online Service, provided by the NASA/Goddard Space Flight Center.

I acknowlegde Doctorate School of Physics, Eötvös University, for supporting my participation on the Gamma-Ray Bursts 2012 Conference - GRB2012, May 07-11, 2012, Munich, Germany.

\section{References}

[1] P. Veres et al., A Distinct Peak-flux Distribution of the Third Class of Gamma-ray Bursts: A Possible Signature of X-ray Flashes?, ApJ 725 (2010) 1955 [arXiv: $1010.2087 \mathrm{v1}$ ]

[2] D. J. Schlegel et al., Maps of Dust Infrared Emission for Use in Estimation of Reddening and Cosmic Microwave Background Radiation Foregrounds, ApJ 500 (1998) 525 [astro-ph/971032 7v2]

[3] Y. C. Pei, Interstellar dust from the Milky Way to the Magellanic Clouds, ApJ 395 (1992) 130 